\title{
Fatigue Test with Stochastic Differential Equation Modeling
}

\author{
E. ERoGlu ${ }^{a}$, I. Guney ${ }^{b}$ And I. Gunes ${ }^{c}$
}

${ }^{a}$ Faculty of Arts and Sciences, Department of Mathematics, Gebze Institute of Technology

Gebze, 41400, Kocaeli, Turkey

${ }^{b}$ Faculty of Arts and Science, Department of Statistics, İstanbul Aydın University, İstanbul, Turkey ${ }^{c}$ Faculty of Engineering, Department of Electrical and Electronics, İstanbul University, İstanbul, Turkey

In this study, a mathematically polymeric insulator material is modeled which is used as a solid insulator in the industry. ASTM D 2303 in inclined plane test method is utilized in order to observe mechanical effect of vibration. The materials have different deformation times before they are fatigued or not fatigued. Our goal is the mathematical model which has materials at progressive times.

PACS: 05.10.Gg, 81.40.Np, 05.45.-a

\section{Introduction}

In electric industry, cheaper and safer transfer of energy is an important issue. Higher voltage is required in order to transport a big amount of energy. The problems facing high voltage are discharge events as well as safety and environmental factors. In industry, solid insulators are employed more often than gas or liquid ones, as they are more resistant in comparison with gas and liquid. A good dielectric material has to possess inevitably, a puncture, low dielectric loss, high mechanical crucible, solidity, moisture and resistance to chemical interaction and temperature. It is also expected to be protected against environmental conditions.

We have inspected aging process in two main questions. Firstly, all the samples have the same conditions, and outer conditions are fixed. Samples are exposed to mechanical vibration with the help of a vibration engine. As the vibration velocity changes, aging effect on the polymeric sample test is inspected. Secondly, all samples are produced under the same conditions and are fatigued before getting age analysis. Corruption is observed in the upcoming fatigue process for pre-experimental fatigue. Now we can introduce our mathematical model concerning aging process in polymeric insulator material.

Stochastic ordinary differential equations (SODEs) offer a convenient and tractable way of modeling the dynamics of many stochastic systems including cracking, fatigue and engineering. One of the most important steps in the modeling process is parameter estimation. However, most data are observed at discrete time frequencies whereas SODEs are almost continuous processes. This introduces "discretization-bias" into estimates which are difficult to eliminate.

An equivalent driving force (EDF) model is proposed for the correlation and prediction of crack growth un- der different stress ratio values $R$. This force defines a completely-reversed stress intensity range that is expected to yield the same crack growth rate as a given stress intensity range $\Delta K$ and stress ratio $R$ [1]. Constitutive equations for the description of failure for cyclic loading of an adhesive interface have been developed within the framework of the cohesive surface methodology [2].

In this study, fatigue crack growth rate in mixed-mode overload (modes I and II) induced a retardation zone that has been predicted by using an "exponential model". The important parameter of this model is the specific growth rate. This has been correlated with various crack driving parameters such as stress intensity factor range, maximum stress intensity factor, and equivalent stress intensity factor, as well as material properties such as modulus of elasticity and yield stress [3].

To study the fatigue crack growth problems and to emphasize the variability of the growth curves in addition to their average growth trend, three stochastic fatigue crack growth models are presented [4].

\section{Model-fatigue analysis by stochastic differential equation}

To illustrate various aspects of the simulation of a time discrete approximation of an Ito process we shall examine a simple example in some detail. We shall consider the Ito process $X=\left\{X_{t}, t \geq 0\right\}$ satisfying the linear stochastic differential equation

$$
\mathrm{d} X_{t}=a X_{t} \mathrm{~d} t+b X_{t} \mathrm{~d} W_{t}
$$

for $t \in\left[t_{0}, T\right]$ with the initial value $X_{0} \in \mathfrak{R}^{1}$. This is an Ito process with drift $a(t, x)=a x$ and diffusion coefficient $b(t, x)=b x$. We know that Eq. (1) has the explicit solution 


$$
X_{t}=X_{0} \exp \left(\left(a-\frac{1}{2} b^{2}\right) t+b W_{t}\right)
$$

for $t \in\left[t_{0}, T\right]$ and the given Wiener process $W=$ $\left\{W_{t}, t \geq 0\right\}[5]$. Knowing the solution (2) explicitly gives us the possibility of comparing the Euler approximation with the exact solution and calculating the error. Consistent estimators for $\alpha$ and $\beta$ are given by Tsay [6], among many others.

\section{Application}

Our data is categorized within low, medium and high vibration levels fracture times and each of these categories is jointly handled. The growth model can be considered as the solution of the stochastic differential equation. In this case, the model explains $88 \%$ of the variance in fracture time. $F$ test $(P<0.001)$ indicates that the independent variable is significantly different from zero. So, we propose our model as follows:

$$
\ln y=0.01 x+3.224 \text {. }
$$

This growth model is obtained for the first three levels (low, medium and high). When the same data are applied to nonlinear regression methods, we find that the magnitude of coefficients are $a=3.22$ and $b=0.01$.

TABLE I

Parameter estimates (for low, medium, high levels).

\begin{tabular}{c|c|c|c|c}
\hline \hline \multirow{2}{*}{ Parameter } & \multirow{2}{*}{ Estimate } & \multirow{2}{*}{ Std. error } & \multicolumn{2}{|c}{$95 \%$ Confidence interval } \\
\cline { 4 - 5 } & & & Lower bound & Upper bound \\
\hline$a$ & 3.224 & 0.025 & 3.170 & 3.279 \\
$b$ & 0.010 & 0.001 & 0.008 & 0.012 \\
\hline
\end{tabular}

Correlation of parameter estimates is -0.897 .

TABLE II

ANOVA (for low, medium, and high levels).

\begin{tabular}{l|c|c|c}
\hline \hline \multicolumn{1}{c|}{ Source } & Sum of squares & $\mathrm{d} f$ & Mean squares \\
\hline regression & 14664.743 & 2 & 7332.371 \\
residual & 23.286 & 13 & 1.791 \\
uncorrected total & 14688.029 & 15 &
\end{tabular}

Parameter estimates (fatigue level).

TABLE III

\begin{tabular}{c|c|c|c|c}
\hline \hline \multirow{2}{*}{ Parameter } & \multirow{2}{*}{ Estimate } & \multirow{2}{*}{ Std. error } & \multicolumn{2}{|c}{$95 \%$ Confidence interval } \\
\cline { 3 - 5 } & & & Lower bound & Upper bound \\
\hline$a$ & 4.601 & 1.568 & -0.390 & 9.591 \\
$b$ & -0.064 & 0.046 & -0.210 & 0.082
\end{tabular}

The nonlinear regression model can be considered as a private solution of black holes (geometric Brownian) differential equation. Then the private solution is

$$
y=\mathrm{e}^{a+b x} .
$$

In a nonlinear regression model, the magnitude of coefficients are $a=3.22$ and $b=0.01$. Table I shows both parameter estimates and confidence intervals for the nonlinear regression model.
ANOVA (fatigue level).

TABLE IV

\begin{tabular}{l|c|c|c}
\hline \hline \multicolumn{1}{c|}{ Source } & Sum of squares & $\mathrm{d} f$ & Mean squares \\
\hline regression & 598.581 & 2 & 299.290 \\
residual & 24.924 & 3 & 8.308 \\
uncorrected total & 623.505 & 5 &
\end{tabular}

TABLE V

Simulation of coefficient of the Brownian differential equation.

\begin{tabular}{c|c|c}
\hline \hline Iteration $(N)$ & $\theta_{1}$ & $\theta_{2}$ \\
\hline 1000 & 1.550443 & 3.211485 \\
5000 & 0.010000 & 3.222309 \\
10000 & 0.010000 & 3.235877
\end{tabular}

Table II demonstrates that both models fit and how much of the residuals are explained by the variables in the nonlinear regression model. It can be observed from Table II that the sum of square (14664.743) and mean square residuals (7332.371) explained by the nonlinear regression model are significantly higher than the unexplained part (23.286). Accordingly, there is no reason to reject the model.

When we apply nonlinear regression estimation to fatigue data, we get the parameter estimates as follows. Table III demonstrates appropriate parameters and Table IV shows the appropriateness of the regression model of these data.

Since the values $a$ and $b$ that correspond to this solution are known, the simulation of the Ito differential equation could be performed. Figure 1 introduces time-vibration trajectories of the performed simulation.

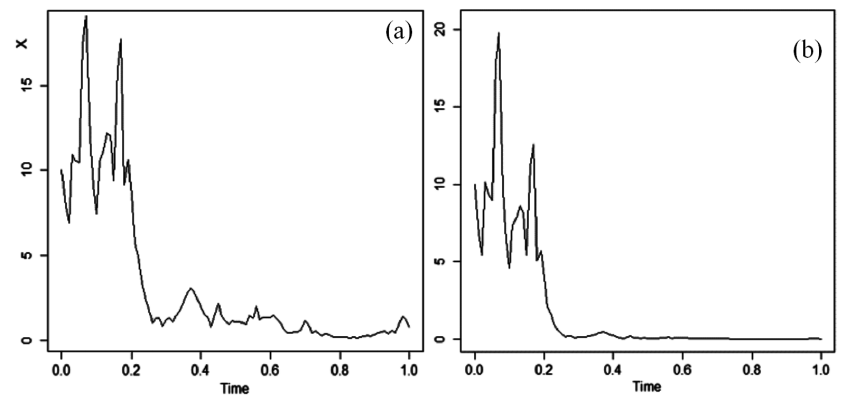

Fig. 1. (a) Geometric Brownian motion trajectory for $b=0.01, a=3.22$; (b) geometric Brownian motion trajectory for $b=-0.064, a=4.601$.

The independent variable analyzed in the previous literature was the fracture size based on fatigue data and the Paris-Erdogan modeling. However, different from previous literature, we seek to understand the role of vibrations on fracture size by using nonlinear regression and the Ito differential equations. Scattered data are seen in Fig. 2 and simulation of coefficient of the Brownian differential equation is presented in Table $\mathrm{V}$. 


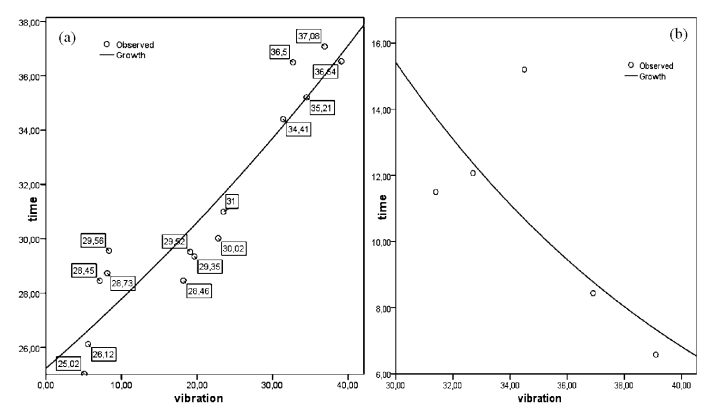

Fig. 2. (a) Graph of model for data; (b) graph of model for fatigue data.

In the simulation above, we empirically show that the convergence of the maximum likelihood estimators to the true parameter values as $N$ increases.

\section{Conclusion}

In this study, the relationship between vibration parameters and duration of use of insulators has been investigated. We have conducted a laboratory experiment in which polymeric insulators were exposed to low, medium, and high vibration levels. The result of such an exposure indicates that as the degree of vibration exposure to such insulators increases, the hazard crack rates of polymeric insulators decreases, hence, survival rate of such insulators increases. On the other hand, we have observed the inverse relationship between vibration exposure and duration of crack rates for fatigue data. Such inverse relationship is due to microcracks found in the polymeric insulators.

\section{References}

[1] S. Kwofie, N. Rahbar, Int. J. Fatigue, 2011.

[2] K.L. Roe, T. Siegmund, Eng. Fract. Mech. 70, 209 (2003).

[3] J.R. Mohanty, B.B. Verma, P.K. Ray, Eng. Fract. Mech. 75, 3941 (2008).

[4] W.F. Wua, C.C. Ni, Probabil. Eng. Mech. 19, 247 (2004).

[5] P.E. Kloeden, E. Platen, H. Schurz, Numerical Solution of Stochastic Differential Equation through Computer Experiments, Springer, Berlin 1994.

[6] R. Tsay, Analysis of Financial Time Series, Wiley Series in Probability and Statistics, Wiley, New York 2002. 